\title{
STABILITY OF ARSININE
}

\author{
Roberto Salcedo* and Lioudmila Fomina. \\ Instituto de Investigaciones en Materiales, UNAM, Circuito Exterior s/n, Ciudad Universitaria, Coyoacán \\ 04510, México D.F. \\ E-mail : dpoliim@servidor.unam.mx
}

\begin{abstract}
The substitution of certain active groups in some positions of the arsinine ring are analyzed from a theoretical point of view. The results show it is possible to find stable arsinine analogues by raising the electronic flow in the ring and, more importantly, straining the ring in order to modify the $\mathrm{C}-\mathrm{As}-\mathrm{C}$ angle.
\end{abstract}

Keywords: Arsinine, Theoretical Calculations, Aromaticity.

\section{Introduction}

Arsinine, arsenic analogue of pyridine, is a relatively unstable molecule, both labile and sensitive to air. However, it is moderately aromatic [1], therefore it should be possible to reinforce its intrinsic aromaticity in order to stabilize the ring, yielding a heterocyclic species useful in organic synthesis.

Research is very limited in the case of heterocycles containing $\mathrm{As}, \mathrm{Sb}$ and $\mathrm{Bi}$, the main reason being their low or null stability. In other words, arsinine can be obtained but it is an air-sensitive compound; stibine is very unstable and spontaneously polymerizes, and bismutine does not exist [2]. Therefore, the available information on these kinds of molecules is very limited.

In the case of arsinine, our main goal is to look for a way of thermodynamically stabilizing the molecule since it is present the best means to find a heavy stable pnicogen heterocycle. This feature has been successfully probed by several strategies, the first of which was the interaction of arsinine itself with transition metal atoms. An arsinine analogue known as cromocene, prepared by Ashe and his collaborators, showed acceptable stability [3]. It seems that the molecule can be stabilized via the electron dispersion from the rings to the metal center. However, this situation requires a good $\pi$ electron current and the concomitant improvement of aromaticity.

One possible pathway for the stabilization of arsinine could be the substitution of specific active groups on the arsinine ring thinking the improvement of the aromaticity can collaborate to decrease the formation enthalpy of this compound. These mentioned groups can activate or deactivate the ring via changes in the electronic flow. This effect depends on the effect they produce on the ring current, in some cases the substituent can release electron density and this feature collaborates to improve the electronic current and the molecule becomes a more thermodynamically species.

In particular, phenyl aromatic rings have been used as substituents. Daly [4] successfully synthesized the 2,5,6 derivative and obtained good quality crystals that were analized by means of X-ray analyses. In the same sense, LeFloch [5] carried out the synthesis of a similar compound containing diphenylphosphines as substituents (see Figure-1). In this case, the large stability of the compound allows them 
to obtain the corresponding crystal form that gives rise to the X-ray description. It should be noted that the authors followed a different synthetic route.

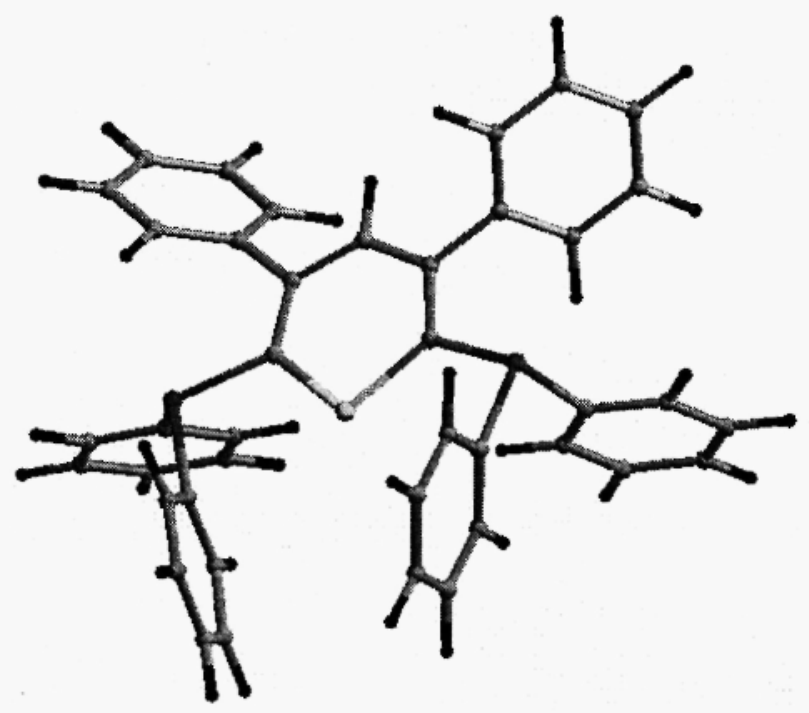

Figure-1 : Compound prepared by LeFloch [5].

The magnitude of the C-As-C angle is a feature to be considered as one of the causes of the stabilization. It has been observed that the C-E-C angle (where $E$ accounts for the pnicogen heteroatom) has a progressive deformation on going from pyridine to bismutine with a tendency to close. This deformation produces a lack of regularity on the dimensions of the rings with the associated lack of aromaticity [1]. It is important to consider that this angle in pyridine is $117.5^{\circ}$, very near to the regular $120^{\circ}$ in benzene. In the case of the molecules prepared by Daly and LeFloch, this angle tends to open, reaching a value similar to that corresponding to phosphinine $\left(100.5^{\circ}\right.$ for the substituted arsinines and $100.2^{\circ}$ for phosphinine [1]). This feature is in direct relationship with the "aromatization" of the substituted molecules.

The aim of this work is to study from a theoretical perspective the nature of the substituents that can improve the aromaticity of arsinine and the role played by the angle $\mathrm{C}$-As-C in the stabilization of the molecule.

\section{Computational Methods}

All calculations have been performed with the Gaussian 98 program [6]. Full geometry optimisations without symmetry constraints were carried out using density functional (DFT) calculations. Becke's gradient corrections [7] for exchange and Perdew-Wang's for correlation [8a] were used for optimisation and total energy evaluation. The correspondent functional is BPW91, which has shown excellent performance in this kind of systems $[8 b, c]$. All calculations were performed using the 6-31$\mathrm{G}^{* *}$ basis set. 
NICS (Nucleus Independent Chemical Shifts) is a technique proposed by Schleyer [9] as an aromaticity index. Following this methodology, we obtained the absolute magnetic shielding at the centre of the rings, which is determined by the non-weighted mean of the heavy atom coordinates, these calculations were carried out at $6-31+G^{*}$ level as it recommended by Schleyer.

\section{Results and Discussions}

The model molecule is shown in Figure-2. The cases under study correspond to the hypothetical molecules that bear the $\mathrm{R}$ substituents shown in the same figure.

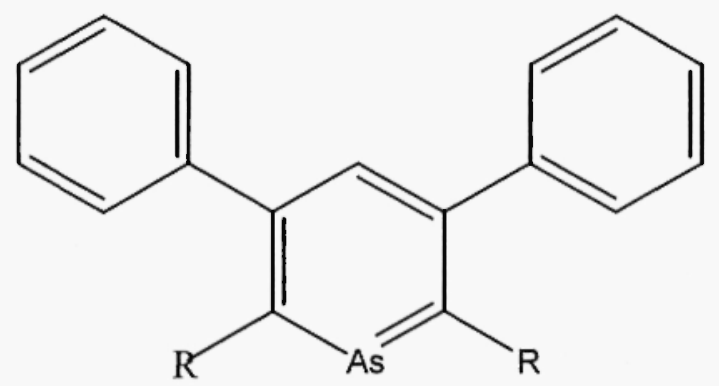
1. $\mathrm{R} 1=-\mathrm{PPh}_{2}$
5. $\mathrm{R} 5=-\mathrm{O}-\mathrm{CH}_{3}$
2. $\mathrm{R} 2=-\mathrm{OH}$
6. $\mathrm{R} 6=\mathrm{CH}_{2}-\mathrm{OH}$
3. $\mathrm{R} 3=-\mathrm{Pyr}$
7. $\mathrm{R} 7=-\mathrm{CH}_{3}$
4. $\mathrm{R} 4=-\mathrm{N}\left(\mathrm{PhCH}_{3}\right)_{2}$
8. $\mathrm{R} 8=-\mathrm{NH}_{2}$

Figure-2 : Molecules under study.

The first case is indeed the LeFloch's molecule. In the other cases, different radicals with electron releasing capability were chosen; the reason for this election is that it is desired to improve the electronic flow within the rings. Compound 1 is the same compound prepared by LeFloch and his group; compounds 2, 7, and 8 bear classical electron releasing groups (that are considered activant groups for electrophilic aromatic substitution by themselves). Compounds 3, 4, 5 and 6 are bulkier groups that, in some cases, can develop intrinsic electronic delocalization or at least some kind of electronic flow, furthermore it has been demonstrated by the successfully synthesis of LeFloch that bulky substituents can help to the stabilization because they hinder a decomposition reaction sterically.

Our hypothesis can be state as follows: the larger the aromaticity, the larger the stability; therefore, a bigger possibility of preparing and isolating a particular arsinine derivative. One of the important features in this study is the role played by the C-As-C angle. It has been observed that when this angle acquires larger values more stable species are found. It is important to highlight that the maximum angle to find in a hexahedral molecule is $120^{\circ}$ as in benzene. The same angle for pyridine is $117.5^{\circ}$; however, in stable phosphinine it is $100.5^{\circ}$ as mentioned above. Thus it is expected that the most stable molecules bear angles near the "magic" number of $120^{\circ}$. 
In the present study, the strategy is to establish a comparison between this angle in the optimized structure and the aromaticity index evaluated by NICS as a stability criteria, this comparison is shown in Table-1 in which the dihedral angle of the heterocyclic ring is included in order to evaluate its planarity in each molecule.

The results show a behavior completely opposite to that expected. The closer the angles, the better the aromaticity values, therefore it is necessary to look for a different explanation.

Obviously, the nature of the substituent is fundamental to explain this behavior. All the molecules that support a substituent that contains an oxygen or nitrogen atom present good aromaticity values and a small C-As-C angle; thus, the explanation should be more complicated than the release or withdrawal nature of the lateral group.

Analyzing Table-1 one can see that aromaticity increases when the substituent has a $2 p$ lone electron pair (cases 2, 5 and 8). It is well known that aromatic molecules should satisfy the Huckel aromaticity rule $(4 n+2)$ [2]. In the case of molecules containing As, one of the electrons is on a $4 p$ As orbital and its delocalization is hampered by the energy and size difference with the $2 p$ orbitals of the carbon atoms. Therefore, the effective number of $\pi$-electrons in molecules containing As is less than 6 , thus, decreasing aromaticity. Substituents bearing $\mathrm{O}$ or $\mathrm{N}$ atoms able to share their $\mathrm{p}$ electrons increase the effective number of $\pi$-electrons in ring containing As, thus increasing their aromaticity (Figure-3)

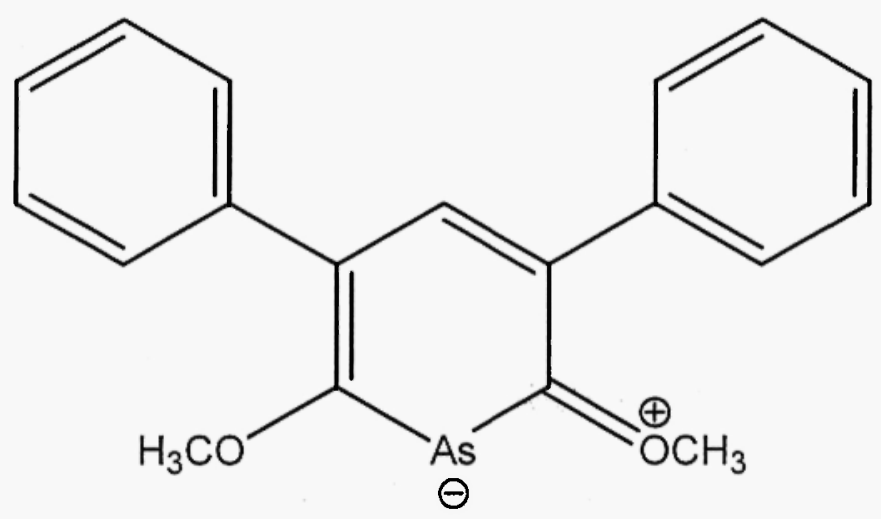

Figure-3 : The resonant structure for $\mathrm{O}$ - and $\mathrm{N}$-containing substituents.

Comparing aromaticity with geometric parameters (see Table-1), one can see that $\mathrm{OCH}_{3}, \mathrm{OH}$ and $\mathrm{NH}_{2}$ substituents favor planarity in agreement with our hypothesis. Molecule 1, bearing $\mathrm{PPh}_{2}$ side groups, is unable to effectively share $p$ electrons with the aromatic ring due to the different energy and size of $2 p$ and $3 p$ orbitals. Since the $\mathrm{C}-\mathrm{C}$ bond is much shorter compared to the $\mathbf{C}$-As one, the planarity increases with a decrease on the C-As-C angle in agreement with the data listed in Table-1. 
Table-1 : Aromaticity and optimize angle

\begin{tabular}{cccc}
\hline Compound & Aromaticity & C-As-C angle & Tor. angle \\
\hline 1 & -5.15 & 99.6 & 0.5 \\
2 & -7.54 & 98.5 & 0.04 \\
3 & -5.8 & 100.5 & 1.3 \\
4 & -5.4 & 101.2 & 2.1 \\
5 & -8.36 & 97.4 & 0.001 \\
6 & -6.84 & 98.7 & 0.3 \\
7 & -5.27 & 101 & 1.8 \\
8 & -6.95 & 99.5 & 1.6 \\
\hline
\end{tabular}

Schleyer has suggested [10] that the angle strain could be negligible if it helps to preserve the planarity of the ring. This is the same in the present case, table 1 shows the torsion angle and it is possible to appreciate that the molecules with the best value of aromaticity are those that remain almost planar. The apparent contradiction is that molecule 4 shows low aromaticity in spite of the presence of nitrogen lone pair. This is related to the fact that $2 p$ lone pair of nitrogen is out of the plane due to steric hindrances caused by the bulky tolyl groups on the one hand and the low availability of this pair due to conjugation with the phenyl rings on the other.

\section{Conclusions}

The possibility of synthesize stable arsinine derivatives is ruled by the nature of the substituent groups it contains. The better option includes several groups that contain oxygen or nitrogen because of their ability to share $2 \mathrm{p}$ electrons that improves the aromaticity value. The opening of the angle C-As-C does not affect stabilization but its closing of helps to preserve the necessary planarity on the respective rings.

\section{Acknowledgements}

We are grateful to Ms. Gabriela Salcedo and Ms. Sara Jimenez for technical assistance.

\section{References}

1. R. Salcedo, J. Mol. Struct. Theochem. 674, 125-129 (2004)

2. P.J. Garrat, “Aromaticity”, Wiley, New York, (1986).

3. Eischenbroich, Ch., Kroker, J., Massa, W., Wünsch, M. \& Ashe, A.J. Ill, Angew Chem. Int. Ed. Engl. 25, 571 (1986).

4. a). Daly, J.J. and Sanz, F., Angew Chem. Int. Ed. Engl., 11, 630 (1972); b). Daly, J.J. \& Sanz, F., J. Chem. Soc. Dalton Trans., 511 (1973).

5. Avarvari, N., LeFloch, P., Ricard, L. \& Mathey, F., Organometallics, 16, 49 (1997).

6. M.J. Frisch, G.W. Trucks, H.P. Schlegel, G.E. Scuseira, M.A. Robb, J.R. Cheeseman, V.G. Zakrzewski, J.A. Montgomery, Jr., R.E. Stratmann, J.C. Burant, S. Dapprich, J.M. Millam, A.D. Daniels, K.N. Kudin, M.C. Strain, O. Farkas, J. Tomasi, V. Barone, M. Cossi, R.Cammi, 
B. Menucci, C. Pomelli, C. Adamo, S. Clifford, J. Ochterski, G.A.Petersson, P.Y. Ayala, K. Morokuma, D.K. Malick, A.D. Rabuck, K.Raghavachari, J.B. Foresman, J. Cioslowski, J.V. Ortiz, B.B. Stefanov, G. Liu, A. Liashenko, P. Piskorz, I. Komaromi, R. Gomperts, L.R. Martin, D.J. Fox, T. Keith, M.A. Al-Laham, C.Y. Peng, A. Nanayakkara, C. Gonzalez, M. Challacombe, P.M.W. Gill, B.G. Johnson, W. Chen, M.W. Wong, J.L. Andres, M. HeadGordon, E.S. Replogle and J.A. Pople Gaussian 98, Gaussian Inc., Pittsburgh, PA, (1998).

7. A.D. Becke, Phys. Rev. A. 38, 3098 (1988).

8. a). J.P. Perdew \& Y. Wang, Phys. Rev. B, 45, 13244 (1992); b). C.J. Wu, L.H. Yang, L.E. Fried, J. Quenneville \& T.J. Martinez, Phys. Rev. B, 67, 235101 (2003); c). Ch. W. Bausclicher, J.W. Lawson, A. Ricca, Y. Xue \& M.A. Ratner, Chem. Phys. Lett., 388, 427 (2004).

9. P.v. R. Schleyer, C. Maerker, A. Dransfield, H. Jiao, \& N.J. R. v. E. Hommes, J. Am. Chem. Soc. 118, 6317 (1996).

10. P.v. R. Schleyer, H. Jiao, H.M. Sulzbach, \& H.F. Schefer III, J. Am. Chem. Soc., 118, 2093 (1996).

Received on April 10, 2006 